\title{
Nefoussa (Djebel) / Adrar n Infusen : Langue et révolution (Témoignage)
}

\section{S. Chaker et Masin Ferkal}

\section{(2) OpenEdition}

\section{Journals}

\section{Édition électronique}

URL : https://journals.openedition.org/encyclopedieberbere/2699

DOI : $10.4000 /$ encyclopedieberbere.2699

ISSN : 2262-7197

Éditeur

Peeters Publishers

\section{Édition imprimée}

Date de publication : 4 octobre 2012

Pagination : 5369-5377

ISBN : 978-90-429-2640-0

ISSN : 1015-7344

Référence électronique

S. Chaker et Masin Ferkal, « Nefoussa (Djebel) / Adrar n Infusen : Langue et révolution (Témoignage) », Encyclopédie berbère [En ligne], 33 | 2012, document N29, mis en ligne le 15 mars 2021, consulté le 17 février 2022. URL : http://journals.openedition.org/encyclopedieberbere/2699 ; DOI : https://doi.org/ 10.4000/encyclopedieberbere.2699

Ce document a été généré automatiquement le 17 février 2022.

(c) Tous droits réservés 


\section{Nefoussa (Djebel) / Adrar n Infusen : Langue et révolution (Témoignage)}

\section{S. Chaker et Masin Ferkal}

1 L'Encyclopédie berbère se tient habituellement à distance de l'actualité immédiate, l'absence de recul ne permettant pas une analyse sereine des faits et situations et de proposer aux lecteurs un état des connaissances solidement documenté, sinon assuré. Même en matière de productions et d'acteurs culturels contemporains, l'EB adopte une approche prudente et mesurée : seules les "valeurs sûres »-produits et producteurs culturels dont le rôle et l'action sont largement reconnus et incontournables dans les sociétés berbères actuelles - ont été et seront traités.

2 Les récents événements de Libye, qui ont abouti à la chute de Kadhafi, ont mis en lumière le rôle considérable des Berbères, notamment ceux du Djebel Nefoussa, dans la lutte contre le régime. Sur le «front de l'Ouest » (Tripolitaine), l'engagement militaire des Berbères semble avoir représenté une contribution décisive à l'effondrement final du régime et à la prise de Tripoli. On laissera aux experts de la chose militaire et aux journalistes le soin d'établir le détail tactique et logistique du dossier, mais ces événements ont mis en lumière un engagement extrêmement vigoureux des Berbères libyens en faveur de la défense de leur identité linguistique et culturelle. Ce qui est une donnée qui ne peut laisser indifférente l'Encyclopédie berbère, d'autant que le phénomène était jusque-là très peu documenté et même largement ignoré. Seules quelques publications militantes d'exilés berbères libyens, en France et en Angleterre (Ussan, $1982 \rightarrow$; Tifinagh, $1984 \rightarrow$, Tamazgha, $1988 \rightarrow$ ), et des cas de répression antiberbère, à Zouara et dans le Djebel Nefoussa, dont l'ampleur est mal connue, permettaient de supposer l'existence d'un mouvement revendicatif berbère en Libye. On pouvait aussi le soupçonner, en creux, à travers l'insistance permanente du régime et de Kadhafi lui-même à affirmer l'identité arabe de la Libye, y compris avant la conquête arabe : ce point est précisément évoqué et documenté dans notre notice M152b « Mythe d'origine : note complémentaire ", EB XXXII, 2010, p. 5170-5172.

Un de nos collaborateurs et ancien étudiant, Masin Ferkal, a eu l'occasion de se rendre en Libye, dans le Djebel Nefoussa, en juillet, puis en septembre 2011. Il en a rapporté un 
témoignage extrêmement précis et illustré par de nombreuses photos, dont nous publions ci-dessous des extraits pertinents.

Dès le début de la révolte en février 2011, lorsque les Berbères d'Adrar $n$ Infusen se sont joints à l'insurrection, toutes les déclarations officielles des groupes locaux d'insurgés ont été faites en berbère ou ont été traduites de manière simultanée de l'arabe. Les édifices publics symbolisant Kadhafi et son régime furent saccagés, des panneaux en tifinagh et des « $\mathrm{Z}$ » (deuxième consonne et médiane du mot aMaZir, symbole identitaire universellement adopté par les Berbères) apparaissent sur les murs des villes et villages berbères du Djebel Nefoussa.

Lorsque la population prit les armes pour faire face aux des troupes kadhafistes, les premiers véhicules utilisés («pick-up») par les combattants d'Adrar n Infusen portaient tous des inscriptions en tifinagh. Des autocollants imprimés, apposés notamment sur les portières des véhicules, portent la mention: Igrawliyen $n$ Adrar $n$ Infusen (= "Révolutionnaires du Djebel Nefoussa ») en tamazight transcrits en caractères tifinagh, avec l'équivalent en langue arabe en-dessous.

Dès les tous premiers moments de la révolte, nombre de jeunes ont confectionné sur les T-shirts qu'ils portaient la lettre « $\mathrm{Z}$ » en tifinagh, symbole de l'amazighité. Cela pouvait d'ailleurs être observé dans la quasi-totalité des reportages réalisés sur place par les télévisions étrangères comme El-Djazeera. La quasi-totalité des combattants portent des t-shirts et/ou des casquettes avec des symboles amazighs : certains avec le signe « $\mathrm{Z}$ », d'autres avec des drapeaux amazighs au dos, d'autres avec divers textes en tifinagh.

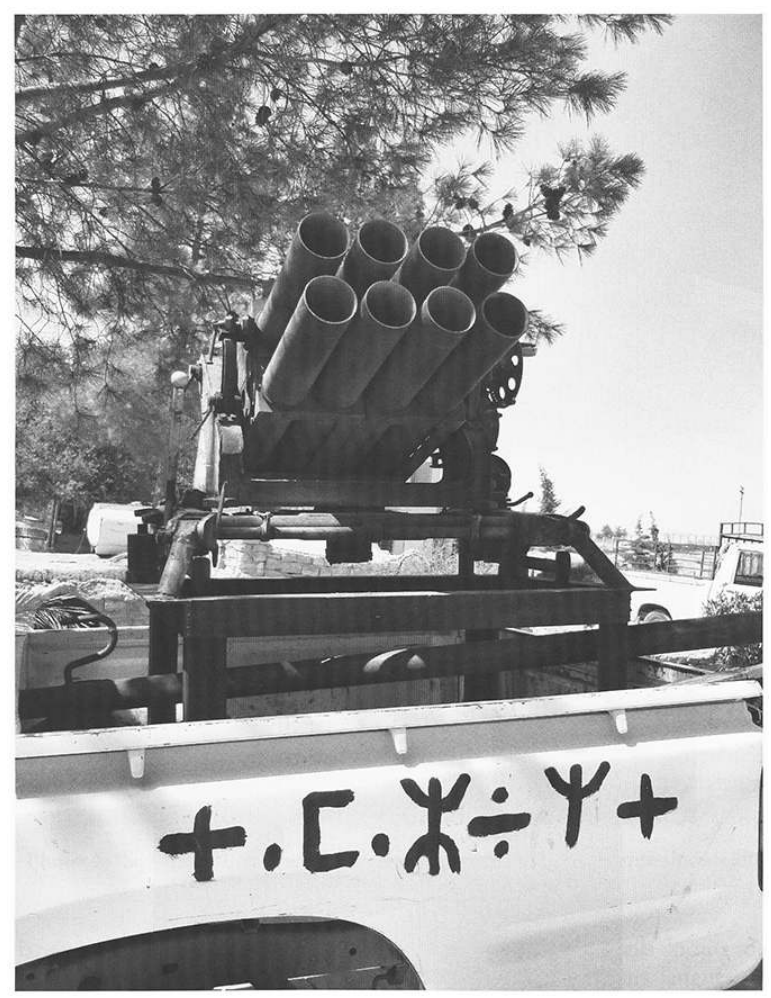

Рното 1 : Un véhicule armé portant différentes inscriptions en tifinagh (Tamazight = « Langue berbère »)

7 Lorsque les troupes kadhafistes furent repoussées des villes et villages d'Adrar $n$ Infusen, la population a pris le contrôle du territoire et a mis en place diverses 
institutions et organisations provisoires. L'expression en langue amazighe s'est immédiatement imposée. Les Conseils locaux de transition (CLT) ont fait de la langue amazighe une de leurs langues de travail. Leurs logos et chartes graphiques portent systématiquement des tifinagh. C'est notamment le cas du CLT d'Ifrane où nous avons séjourné en juillet 2011 ; dans toutes les expressions graphiques des CLT de la région, la langue amazighe est en première position. L'ensemble des documents officiels sont rédigés en tamazight et en arabe. Toujours à Ifrane, sur centre de commandement militaire flotte un drapeau amazigh aux côtés du nouveau drapeau libyen.

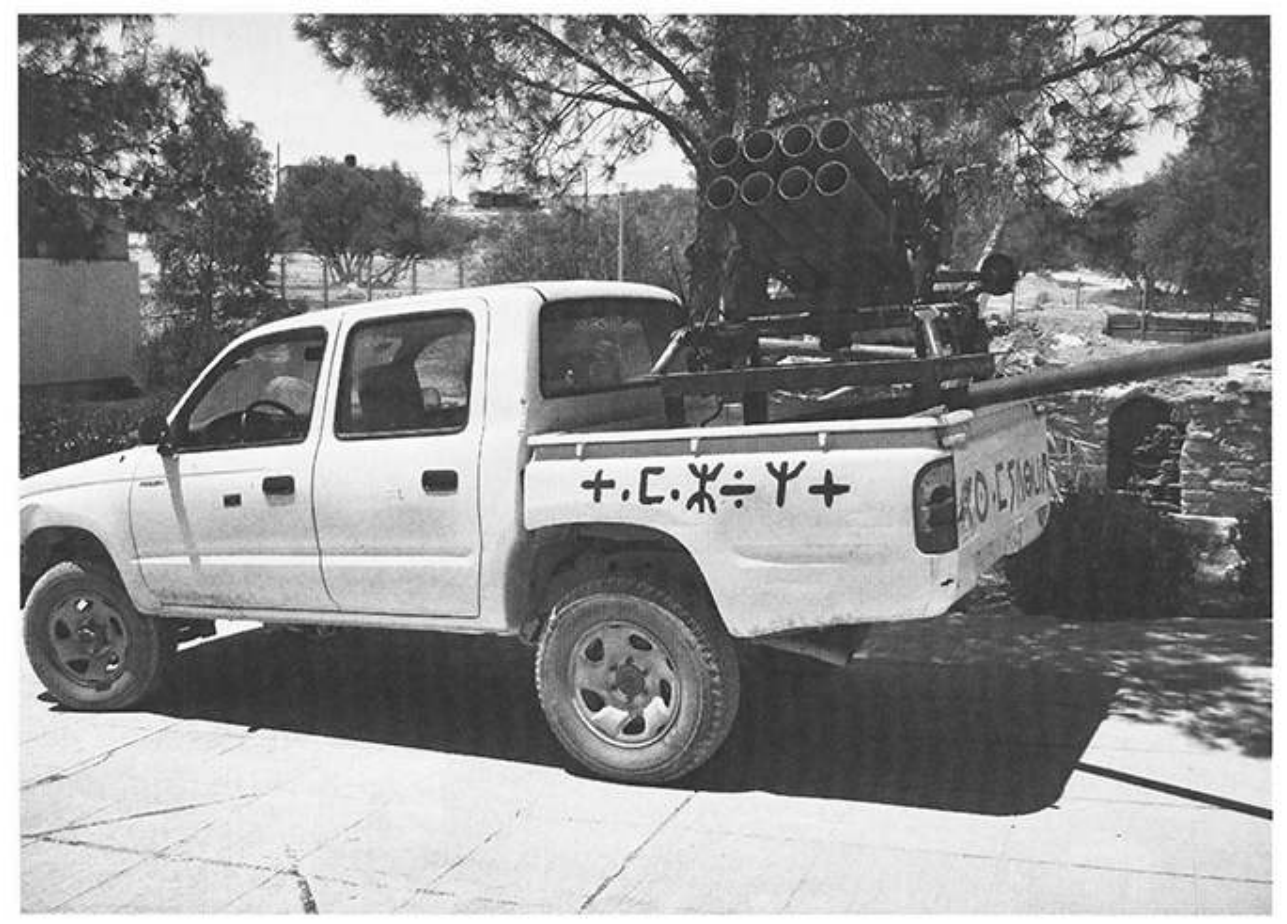

Pното 2 : Un véhicule armé portant différentes inscriptions en tifinagh (Tamazight = « Langue berbère ») 


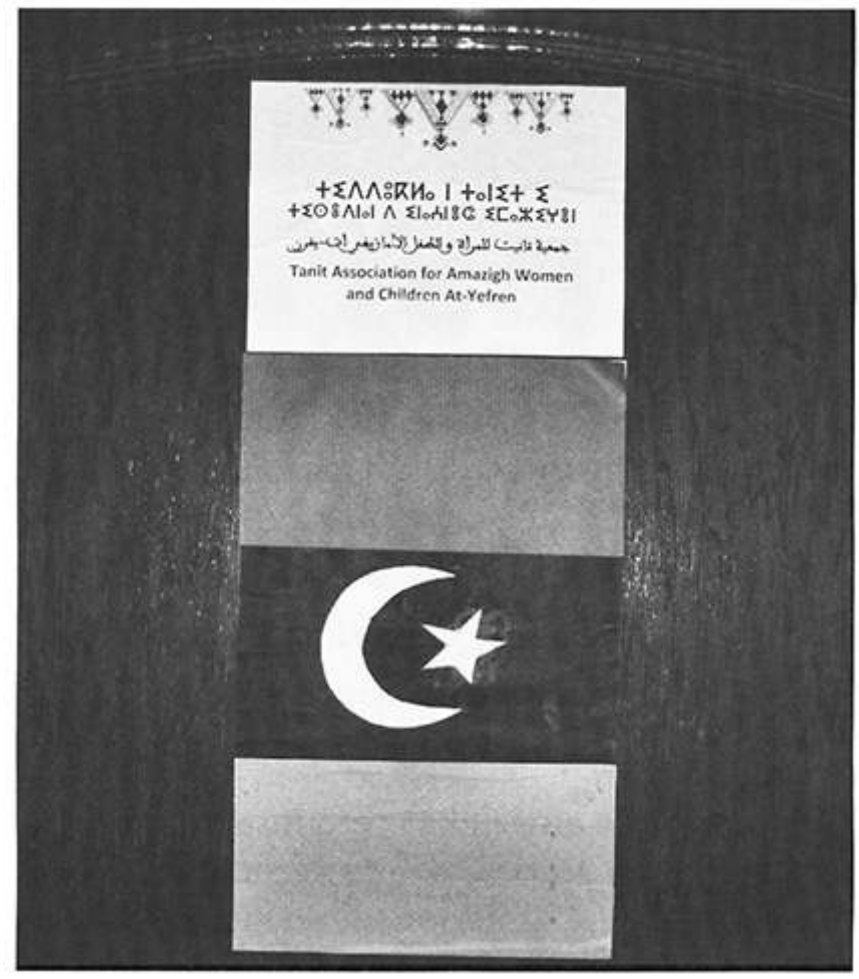

Pното 3 : Signalétique bi- (ou tri-)lingue dans les locaux des Comités locaux du Djebel Nefoussa (Yefren).

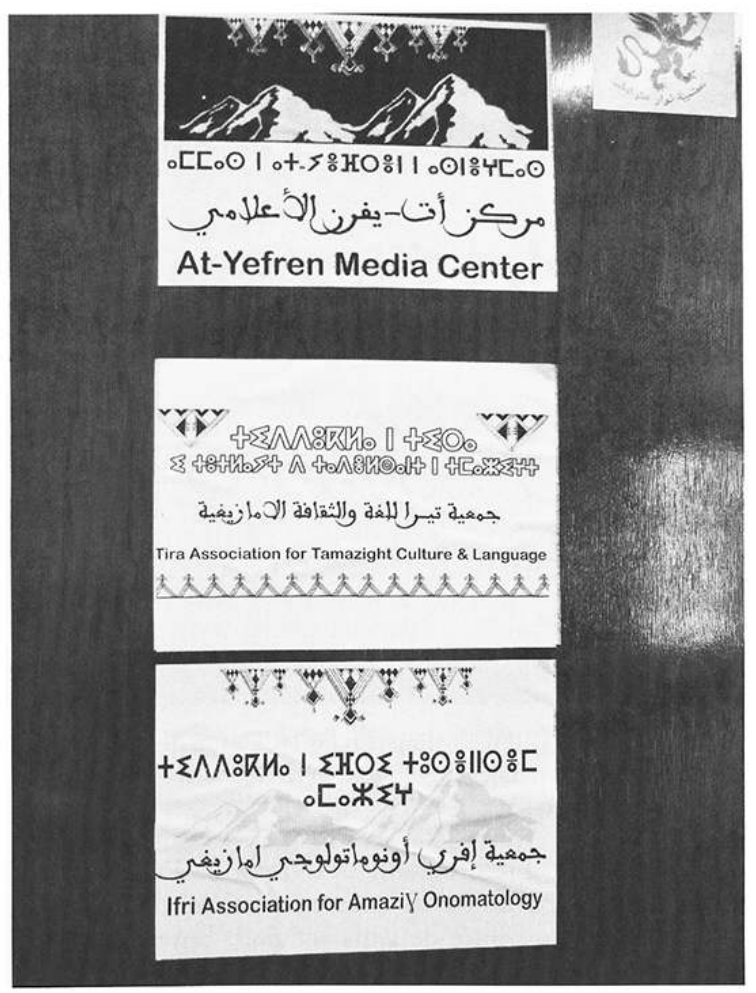

Pното 4 : Signalétique bi- (ou tri-)lingue dans les locaux des Comités locaux du Djebel Nefoussa (Yefren).

8 Dans cette région d'Ifrane, qui regroupe une dizaine de villages, très vite après sa libération, un bâtiment a été utilisé pour accueillir un Centre d'information (Centre 
d'information d'At-Yefren). En plus de son rôle d'information, le Centre abrite diverses associations de la société civile: toutes ont tamazight au centre de leurs activités. Enseignement de tamazight, promotion de l'art amazigh, travail sur la toponymie, etc. Les langues de travail aussi bien du Centre que de ces associations sont conjointement la langue amazighe et la langue arabe. Leurs documents sont systématiquement en tamazight et en arabe et parfois en anglais comme troisième langue. La langue amazighe est toujours placée en première position.

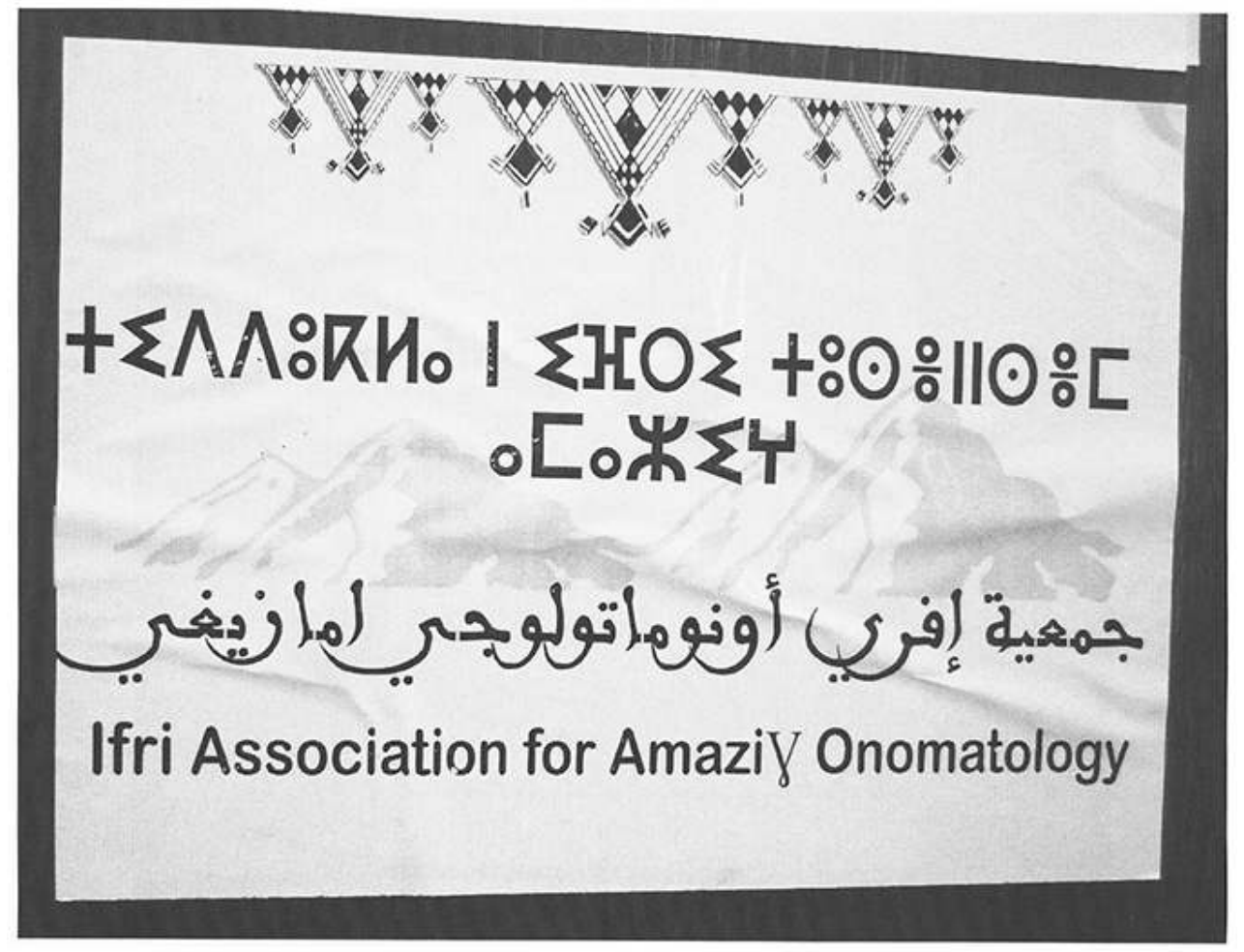

Рното 5 : Signalétique bi-(ou tri-)lingue dans les locaux des Comités locaux du Djebel Nefoussa (Yefren).

9 L'ensemble des espaces publics sont nommés en premier en langue amazighe. C'est le cas, toujours à Ifrane, de cet espace nommé Taddart n Tlelli ("Maison de la Liberté »), ancien espace officiel que le régime de Kadhafi utilisait pour sa propagande. Dans nombre de villes comme Jadu, Ifrane ou Kabaw, des associations enseignent régulièrement tamazight aux enfants.

\section{En Tunisie parmi les réfugiés}

10 On signalera que lors des moments les plus difficiles, au printemps 2011, sous le feu des forces kadhafistes, la langue amazighe a été utilisée et revendiquée aussi bien parmi les combattants en Libye que parmi les nombreux réfugiés en Tunisie. Dès les premières vagues d'arrivants en Tunisie, apparaissaient dans les camps de réfugiés des drapeaux amazighs ou encore des drapeaux libyens dont l'étoile est remplacée par le fameux « $\mathrm{Z} »$. 


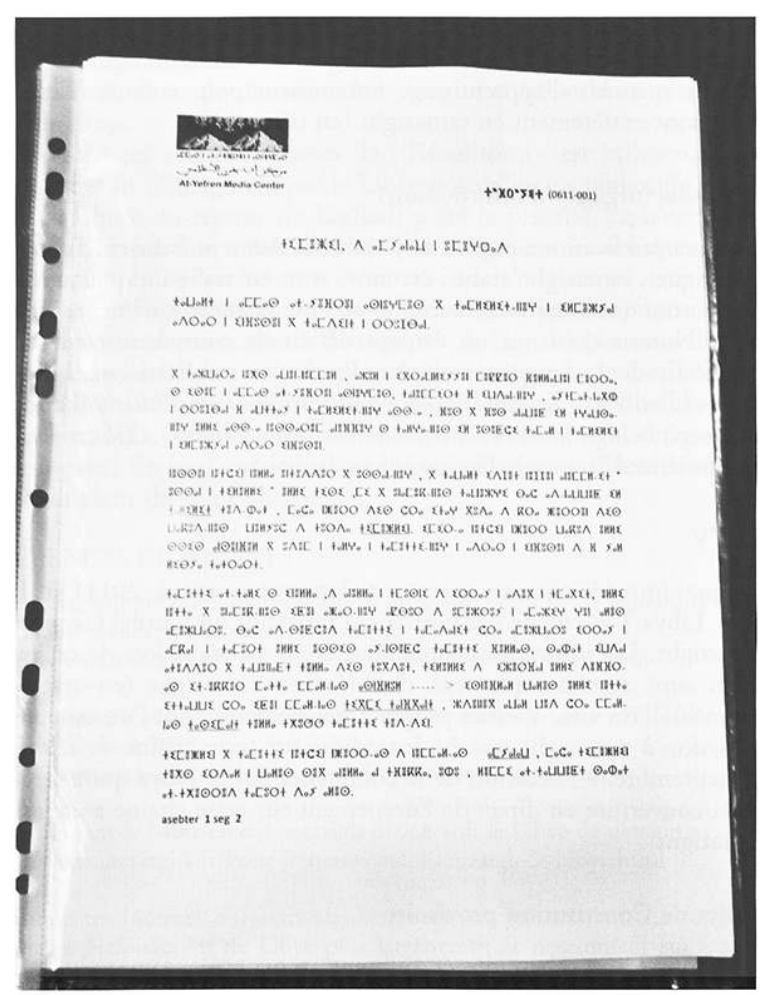

Pното 6: Signalétique bi-(ou tri-)lingue dans les locaux des Comités locaux du Djebel Nefoussa (Yefren).

Photos de l'insurrection berbère du Djebel Nefoussa (juillet, septembre, octobre 2011).

Clichés M. Ferkal

Beaucoup de militants amazighes réfléchissaient dans ces bases de repli à l'avenir de la Libye et de tamazight. Nombre d'entre eux sont originaires de Zwara (At-Wilul). Leur activité principale est la publication du journal Tagrawla ("Révolution») dans les deux langues, tamazight et arabe. Parmi eux, aussi un groupe réfléchit sur l'enseignement de tamazight et confectionne des manuels d'apprentissage notamment pour enfants. Ces petits manuels sont entièrement en tamazight (en tifinagh).

\section{Publications (organes d'information)}

Plusieurs publications ont vu le jour dans Adrar $\mathrm{n}$ Infusen. La plupart sont bilingues, tamazight/arabe ; certaines sont en arabe uniquement, mais les noms sont toujours en tamazight et sont systématiquement écrits en tifinagh. Notons qu'il y a un évident déficit de compétence en matière d'usage écrit de la langue amazighe. Parmi les publications, signalons: Tilelli («Liberté ») Tagrawla («Révolution»), Tamellult ( Blanche »), Ussan nnegh (« Nos jours»), Iderfan (« Hommes libres»), Tifawin $n$ Ifran (« Les lumières d'Ifrane »), Izerfan (« Les droits »)...

\section{Libya TV}

Presque immédiatement après son lancement (avril 2011) à Doha (Qatar), Libya TV, chaîne des insurgés, a introduit un journal (Ineghmisen) en tamazight. Les interventions qui sont faites en arabe lors de ce journal amazigh sont systématiquement doublées en 
tamazight (en traduction simultanée). Très vite, d'autres programmes berbères dont l'un est consacré à l'initiation à tamazight ont été introduits sur cette chaîne de télévision. Le 15 septembre, à l'occasion de la conférence sur la Libye qui a eu lieu à Paris, la couverture en direct de l'événement sur cette chaîne a été assurée en tamazight.

\section{Le projet de Constitution provisoire}

Pour tous les combattants et militants rencontrés, tamazight est une évidence et tous attendent qu'un statut lui soit reconnu dans la nouvelle constitution. Plusieurs conseils locaux de transition, comme celui d'Ifrane, ont fait de tamazight une langue officielle puisque l'ensemble de ses documents sont rédigés en tamazight et en arabe. L'ensemble des associations et organisations civiles d'Adrar $\mathrm{n}$ Infusen demandent la reconnaissance de tamazight comme l'une des langues officielles du pays ainsi qu'en témoignent le document intitulé "La Libye de demain » rédigé par le Mouvement culturel amazigh d'Adrar $\mathrm{n}$ Infusen ou le communiqué des organisations civiles d'Adrar $\mathrm{n}$ Infusen daté du 18 août 2011 qui conteste le projet de déclaration constitutionnelle du Conseil National de Transition ou encore le communiqué du Congrès national amazigh libyen, réuni le 26 août à Tripoli, qui rejette catégoriquement l'article relatif à la langue officielle du projet constitutionnelle du CNT et écarte toute négociation sur la question de l'officialisation de tamazight. L'adoption de l'hymne national libyen en tamazight, entonné sur la Place des Martyrs à Tripoli par des dizaines de milliers de manifestants, vient appuyer cette détermination des Imazighen de donner à leur langue et identité sa place dans la nouvelle Libye.

Pour tous ces acteurs berbères, la « Révolution » est intimement liée à tamazight et ils n'imaginent pas la Libye nouvelle sans tamazight. Et, pour eux, si la chute du régime de Kadhafi a été la priorité, ce n'est pas pour autant que la question amazighe doit être reléguée aux oubliettes.

Le débat qui s'amorce autour de la future constitution pourraient donc être assez houleux, voire source de tensions, le Conseil National de Transition (de Benghazi) étant assez largement dominé par les arabophones et les courants islamistes. La volonté démocratique des anti-kadhafistes libyens ira-t-elle jusqu'à accepter et reconnaître la diversité linguistique et culturelle du pays? En tout cas, les berbérophones libyens semblent bien décidés à défendre leur droit à l'existence. 


\section{Références et sources}

- Le communiqué du Congrès national amazigh, réuni le 26 septembre 2011 est consultable à

l'adresse Internet suivante :

http://www.tamazgha.fr/Le-Congres-national-amazigh-libyen.html

- Communiqué des organisations de la société civile d'Adrar n Infusen réunies à Ifrane le 18 août 2011 :

http://www.tamazgha.fr/Constitution-libyenne-la-societe.html

- «Comment le Mouvement amazigh libyen voit la Libye de demain »:

http://www.tamazgha.fr/Pour-le-mouvement-amazigh-la-Libye.html

Plusieurs sites Internet diffusent régulièrement des informations sur les Berbères du Djebel Nefoussa (et de Libye plus largement), et notamment sur leur luttes récentes et actuelles : http:// tamazgha.fr/

La télévision des opposants au régime de Kadhafi diffuse depuis le printemps 2011 un journal d'informations en berbère :

http://www.livestation.com/channels/125-libya-tv-arabic

Voir aussi :

FERREIRA Davina : La montagne berbère, un refuge pour un peuple nié :

http://base.d-p-h.info/pt/fiches/dph/fiche-dph-7056.html

http://base.d-p-h.info/pt/fiches/dph/fiche-dph-7051.html

Pour un vision d'ensemble sur la « question berbère » en Libye, on se reportera à :

CHAKER S. \& FERKAL M., « Berbères de Libye : un paramètre méconnu, une irruption politique inattendue. », Politique africaine, 125, mars 2012, p. 105-126 (Dossier « La Libye révolutionnaire », coordonnée par Ali Bensaad).

INDEX

Mots-clés : Libye, Nefoussa, Politique 